\title{
Neural Machine Translation using Recurrent Neural Network
}

\author{
Debajit Datta, Preetha Evangeline David, Dhruv Mittal, Anukriti Jain
}

\begin{abstract}
In this era of globalization, it is quite likely to come across people or community who do not share the same language for communication as us. To acknowledge the problems caused by this, we have machine translation systems being developed. Developers of several reputed organizations like Google LLC, have been working to bring algorithms to support machine translations using machine learning algorithms like Artificial Neural Network (ANN) in order to facilitate machine translation. Several Neural Machine Translations have been developed in this regard, but Recurrent Neural Network (RNN), on the other hand, has not grown much in this field. In our work, we have tried to bring $R N N$ in the field of machine translations, in order to acknowledge the benefits of RNN over ANN. The results show how RNN is able to perform machine translations with proper accuracy.
\end{abstract}

Keywords: Neural Machine Translation, Recurrent Neural Network, Long Short-Term Memory, Speech Recognition.

\section{INTRODUCTION}

A normal human being can express as much as eleven thousand to twenty-five thousand words every day. This makes speech the most well-known method for communication and basic method of conveying message that is needed to be conveyed. Be it a discussion, discourse, speech, introductions or any broad talks, we use speech to express contemplations and activities, and the speech need not be in the language known to everyone present there. In a situation where both sides are ignorant of the language used in the exchange, the cycle will be irrelevant. Consequently, a framework is needed that can connect this language boundary. Speech to speech translation is one such framework that can prove to be useful by facilitating the chat between people communicating in various dialects. As seen from this period of worldwide and interconnected economy, information communication has gotten unavoidable. [1] The worldwide situation includes the interest of understanding among speakers of various dialects. Further, the actual task of

Revised Manuscript Received on April, 042020.

* Correspondence Author

Debajit Datta*, B-Tech, Vellore Institute of Technology, Vellore, India. Email: debajit.datta2000@gmail.com

Dr. Preetha Evangeline David, Assistant Professor (Sr. Grade), Vellore Institute of Technology, Vellore, India. Email: preetha.evangeline@vit.ac.in

Dhruv Mittal, B-Tech, Vellore Institute of Technology, Vellore, India. Email: mittaldhruv29@gmail.com

Anukriti Jain, B-Tech, Vellore Institute of Technology, Vellore, India. Email: anukritijainvit@gmail.com

(C) The Authors. Published by Blue Eyes Intelligence Engineering and Sciences Publication (BEIESP). This is an open access article under the CC BY-NC-ND license (http://creativecommons.org/licenses/by-nc-nd/4.0/) spanning the Digital-separation can never be cultivated in genuine sense without breaking the language obstructions with an insightful framework or machines. Speech translation innovation - having the option to talk and have one's words made an interpretation of naturally into the other individual's language - has for quite some time been a fantasy of mankind. Most populations around the globe find acquiring and understanding foreign languages highly difficult because of the obvious geographical reasons, which is why speech to speech translation system in today's world is a great boon.

Speech translation is basically a way to convert the language spoken by a certain individual into another preferable language. Google has its own algorithm that uses Artificial Neural Network (ANN) in order to facilitate machine translation using Neural Machine Translation (NMT), called GNMT (Google NMT). Now, Recursive Neural Network (RNN) can model sequence of data, like time series, so that each sample can be assumed to be dependent on the previous ones, unlike Artificial Neural Network. Also, RNN can even be used with convolutional layers to extend the effective pixel neighborhood. Thus, RNN is preferred comparatively more these days. There exists a great build-up of materials that is needed to be interpreted and analyzed for organizations, instruction, trade, the travel industry and so forth. Innovative help similar to machines' help for this is required.

Neural Network is a kind of artificial intelligence that deals with imitating the way a human brain works. A neural network functions after establishing connections between the elements that are involved in the processing. This connection acts as the neurons in living animals. These connections and imitation of animal neuron are particularly effective for predicting all the events when the networks have quite vast database of prior examples or classification, to draw on. Recursive Neural Network is a kind of neural network that is designed and deployed by applying the same set of weights in a recursive manner over a structured input, to come up with a structured prediction on a random input structure or come up with a scalar prediction on the structured input. This is carried out by letting the machine traverse the given structure in a systematic topological order, where the first step comes first and second step comes later, like in a directed graph if there exists an edge $\mathrm{xy}$ then $\mathrm{x}$ comes before $\mathrm{y}$ in the ordering. Artificial Neural Networks, also called connectionist systems, are the computing systems which can perform tasks by simply learning from other examples, most of the time even without programming with the specific steps for the tasks.

Published By:

Blue Eyes Intelligence Engineering \& Sciences Publication

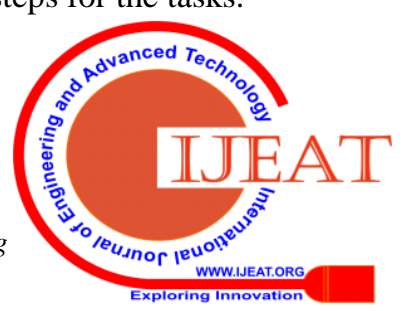


It is based on the collection of connected nodes which try to replicate animal neurons in the biological brain. The artificial neurons are capable of receiving signals from a source or other artificial neurons and then process it, and transmit the signal to the neurons connected to it. Recursive Neural Network (RNN) can model sequence of data, like time series, so that each sample can be assumed to be dependent on the previous ones, unlike Artificial Neural Network (ANN). Also, RNN can even be used with convolutional layers to extend the effective pixel neighborhood. There is a constant backpropagation or backtracking within memory cells in RNN, so it possesses the capability to bridge very long lags in time. Recurrent neural networks are capable of handling noises as well as continuous values. [2] Unlike finite state automata or hidden Markov models, recurrent neural networks require no a priori choice of a finite number of states, as it has an ability to deal with unlimited number of states.

This project aims to create a system that is able to facilitate speech translation among various languages using a three-stage model. These three main modules include Speech Recognition, Machine Translation and Speech Synthesis use different tools and concepts for their specific purpose. Google APIs have been used to convert text to speech and speech to text, while the translation is carried out using Recurrent Neural Network (RNN). The use of RNN for helping in translation is elaborated and further the unique part of speech synthesis is explained in detail. In addition, system architecture has been provided along with the services and communication protocols for connecting the client to the main speech to speech translation servers. The research aims to deal with the underlying pipelined engineering of automated speech acknowledgment, machine translation framework and speech synthesis or content to speech which principally depends on lexical data and disregarding the other rich data which is available in speech and spoken talk, for example, commotion and human articulations. The foundation for the system is that, it focuses on the rich context free of the actual dictated phrases, along with having an understanding of and corresponding with variety of cultural people making the data transfer better, and more efficiency in communication. This project aims to help in understanding the need for avoiding dividing the task of speech to speech translation into various stages, and also in increasing of the inference speed whilst cultivating the speech of the individual with less errors in recognition and translation.

\section{RELATED WORK}

There have been several researches in the field of Recurrent Neural Networks and Machine Translations. Research workers have come up with several methods and approaches in to bring forth the techniques that can really be helpful for machine translations. Several parameters have also been checked by them which can be useful for future development of machine translation systems.

The research by Shadiev et. al. [1] discusses the effectiveness of STR application on students learning performance, during and after the collaborative learning activities on the online synchronous cyber classrooms. The application has been implemented on the open university students, using STR. The usage of STR was implemented among students with cognitive environment, collaborative academic activities, non-native speakers and students. Dataset used in this paper is Windows Speech Recognition in the Microsoft Operating System, IBM ViaVoice software. The paper has used its dataset to be the Windows Speech Recognition in the Microsoft Operating System IBM ViaVoice software. Most experimental students perceived that STR was useful for individual presentations and for essays writing. Students who were exposed to the STR were willing to use STR system for learning in the future. One of the most common concerns reported in relation to online learning literature is the poor audio quality due to restricted internet bandwidth availability. The above-mentioned problems can be solved by adopting some assistive media-to-text recognition technologies, such as writing-to-text, image-to-text, diagram-to-text, text-to-speech, speech-to-text, and handwriting-to-text. The work of Soltau et. al. [2] deals with usage of deep bi-directional LSTM RNNs with CTC loss. Neural Speech Recognizer model has a deep LSTM RNN architecture built by stacking multiple LSTM layers. Since the bidirectional RNN models have better accuracy and their application is offline speech recognition on the system, they have used two LSTM layers at each depth - one operating in the forward and another operating in the backward direction in time over the input sequence. Vocabulary corpus has been used as dataset here, that has 296 videos from 13 categories, with each video averaging 5 minutes in length. The total test set duration is roughly 25 hours and 250,000 words. The output from the CTC layer, essentially making the CTC word model an end-to-end all-neural speech recognition model. The bi-directional LSTM CTC word models are capable of accurate speech recognition with no language model or decoding involved. The error rate calculation disadvantages the CTC spoken word model as the references are in written domain, but the output of the model is in spoken domain, creating artificial errors like "three" vs "3". The entire speech recognizer becomes a single neural network.

According to Xiong et. al. [3], the use of various convolutional and LSTM acoustic model architectures, combined with a novel spatial smoothing method and lattice-free MMI acoustic training, multiple recurrent neural network language modeling approaches, and a systematic use of system combination. They have used CNN, LSTM, Spatial smoothing, speaker adaptive modelling, lattice-free sequence training. The 4-gram language model for decoding was trained on the available CTS transcripts from the DARPA EARS program: Switchboard (3M words), BBN Switchboard-2 transcripts (850k), Fisher (21M), English CallHome (200k), and the University of Washington conversational Web corpus (191M). The only exception is the VGG+ResNet system, which combines acoustic senone posteriors from the VGG and ResNet networks. While this yields our single best acoustic model, 
only the individual VGG and ResNet models are used in the overall system combination. In the included model, N-best output from all systems are combined confusion network construction generates new possible hypotheses not contained in the original N-best lists the machine errors are substantially the same as human ones, with one large exception: confusions between backchannel words and hesitations.

The paper presented by Shang et. al. [4] discusses a general encoder-decoder framework: it formalizes the generation of response as a decoding process based on the latent representation of the input text, while both encoding and decoding are realized with recurrent neural networks (RNN). The dataset consists of a corpus of roughly 4.4 million pairs of conversations from Weibo. They use an encoder-decoder based neural network to generate a response in STC. They have also empirically verified that the proposed method can yield performance better than traditional retrieval-based and translation-based methods. Widely accepted evaluation methods in translation do not apply. It is also not reasonable to evaluate with Perplexity, a generally used measurement in statistical language modeling, because the naturalness of response and the relatedness to post cannot be well evaluated. Natural language conversation is one of the most challenging artificial intelligence problems, which involves language understanding, reasoning, and the utilization of common-sense knowledge. Automatic evaluation of response generation is still an open problem. Similar to the previous works, the research work that has been carried out by Battenberg et. al. [5] discusses empirical comparison among the CTC, RNN-Transducer, and attention-based Seq2Seq models for end-to-end speech recognition. Simplifying speech recognition pipeline so that decoding can be expressed purely as neural network operations. On simplifying speech recognition pipeline so that decoding can be expressed purely as neural network operations. It has been done on Hub5'00 dataset. The choice of the encoder plays a crucial role in optimizing the performance of three models: CTC, RNN-transducer and attention-based seq-seq model. In attempt to train RNN-Transducer models with the streaming constraint, and in reducing computation in encoder layers, it is found that CTC and attention models still have strengths that we aim to leverage in the future work with RNN-Transducers.

In the work of Bahdanau et. al. [6] they have proposed two types of models. The first one is an RNN Encoder-Decoder and another is RNNsearch. The general model proposed for neural machine translation often belongs to a family of encoder-decoders and encode a source sentence into a fixed-length vector from which a decoder generates a translation. Dataset used in this project is Europarl (61M words), news commentary (5.5M), UN (421M) and two crawled corpora of $90 \mathrm{M}$ and $272.5 \mathrm{M}$ words respectively, totaling $850 \mathrm{M}$ words. In this paper they have two types of models. The first one is an RNN Encoder-Decoder and another is RNNsearch. The experiment revealed that the proposed RNNsearch outperforms the conventional encoder-decoder model (RNNencdec) significantly, regardless of the sentence length and that it is much more robust to the length of a source sentence. One of challenges left for the future is to better handle unknown, or rare words. This will be required for the model to be more widely used and to match the performance of current state-of-the-art machine translation systems in all contexts. Luong et. al. [7] in their work, have developed a system that examines two simple and effective classes of attentional mechanism: a global approach which always attends to all source words and a local one that only looks at a subset of source words at a time. They demonstrate the effectiveness of both approaches on the WMT translation tasks between English and German in both directions. Dataset used in this is WMT'14 training data consisting of $4.5 \mathrm{M}$ sentences pairs (116M English words, $110 \mathrm{M}$ German words). Their various attention-based models are classified into two broad categories, global and local. These classes differ in terms of whether the "attention" is placed on all source positions or on only a few source positions. For the English to German translation direction, our ensemble model has established new state-of-the-art results for both WMT'14 and WMT'15, outperforming existing best systems, backed by NMT models and n-gram LM re-rankers, by more than 1.0 BLEU Some functions can be compared on various alignment functions and shed light on which functions are best for which attentional models.

It has been discussed by Cho et. al. [8], that the encoder and decoder of the proposed model are jointly trained to maximize the conditional probability of a target sequence given a source sequence. They show that the proposed model learns a semantically and syntactically meaningful representation of linguistic phrases. The project has been worked out on the dataset Europarl (61M words), news commentary (5.5M), UN (421M), and two crawled corpora of $90 \mathrm{M}$ and $780 \mathrm{M}$ words respectively. The contribution by the RNN Encoder- Decoder is rather orthogonal to the existing approach of using neural networks in the SMT system, so that we can improve further the performance by using, for instance, the RNN Encoder- Decoder and the neural net language model together. Apply the proposed architecture to other applications such as speech transcription. On the contrary, the work of Cho et. al. [9] shows that the neural machine translation performs relatively well on short sentences without unknown words, but its performance degrades rapidly as the length of the sentence and the number of unknown words increase. The project is based on the widely used and implemented dataset, which has been used for testing several models over the years, called Europarl (61M words), news commentary (5.5M), UN (421M) and two crawled corpora of $90 \mathrm{M}$ and $780 \mathrm{M}$ words respectively. A binary convolutional neural network whose weights are recursively applied to the input sequence until it outputs a single fixed-length vector. The performance of the neural machine translation suffers significantly from the length of sentences. However, both models are able to generate correct translations very well. Need to explore different neural architectures, especially for the decoder. Despite the radical difference in the architecture between RNN and grConv which were used as an encoder, both models suffer from the curse of sentence length. The research carried out by Sennrich et. al. [10],

Blue Eyes Intelligence Engineering \& Sciences Publication

(C) Copyriaht: All riahts reserved.

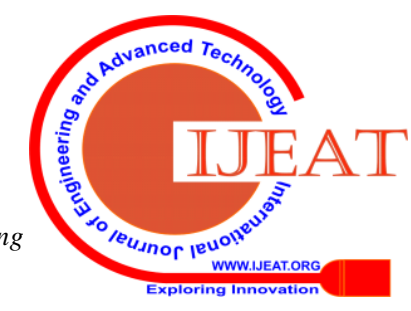


discusses the suitability of different word segmentation techniques, including simple character $\mathrm{n}$ - gram models and a segmentation based on the byte pair encoding compression algorithm.

The neural machine translation system is implemented as an encoder-decoder network with recurrent neural networks. The dataset that has been used in this project is WMT 2015 set consists of 4.2 million sentence pairs, or approx. 100 million tokens.

The neural machine translation systems are capable of open-vocabulary translation by representing rare and unseen words as a sequence of subword units. There is further potential in bilingually informed segmentation algorithms to create more align-able subword units, although the segmentation algorithm cannot rely on the target text at runtime.

\section{PROPOSED SYSTEM}

Artificial Neural Networks have been widely accepted as the base of Neural Machine Translations these days, whereas, Recurrent Neural Networks are not much popular right now for integrating them with the neural machine translation systems. Although ANN comes with several benefits including its ability to generalize and learn and model non-linear and complex relationships, there are some inevitable advantages of RNN over ANN like the fact that unlike ANN, RNN can model sequence of data for instance, the time series - which helps to validate the assumption that each sample is dependent on the previous samples.

There are several android apps and web apps to translate the sentences and paras into different languages but none of them speak on behalf of you in a different language. Even google translator will just take the text and then translate it to a text format. Our project will listen to what you say, translate into different languages and speak up for you when required. To do this we will be using the gTTS (Google Text-to-Speech, a Python library and CLI tool) API and Google Speech Recognition API along with our RNN model for translation (into different languages). Also, at the same time, most of the Web applications or Android applications or iOS applications like Google Translator use Neural Machine Translators that uses ANN (Artificial Neural Network) for the translation process but we will be using RNN (Recurrent Neural Network) in our project. The benefit of using RNN in our project is that it will allow us to work on sequential data i.e. we can have words and sentences in a text which are based on previous context as well. This can't be achieved using ANN.

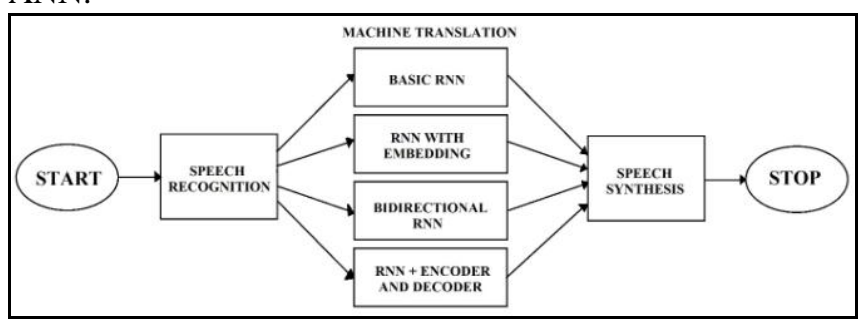

Fig. 1. Flowchart of the system.

The Fig. 1 explains the flow within the several modules that has been implemented in the proposed system.

\section{IMPLEMENTATION}

\section{A. Dataset}

The system helps in translating from English to French, so the dataset that has been used contains English and French sentences. The dataset is taken from an open GitHub source under username 'susanli2016' in the data folder of the project title 'NLP-with-Python', titled 'small_vocab_en' and 'small_vocab_fr'.

Statistically, the English corpus consists of 1,823,250 English words, out of which 227 are unique English words. In the French corpus, there are 1,961,295 French words, out of which 355 are unique French words.

\section{B. Modules}

The system has been broadly divided into three distinct modules, the one where the input speech is recognition and interpreted, the output of the first module is further given as input to the next module which is Machine Translation where the main translation takes place, and final module is the one where new speech is synthesized on the basis of output given from the Translation module.

The first module is Speech Recognition. This module deals with the conversion of spoken data into the corresponding text. The text which is generated after interpretation of the spoken data, becomes the output of this module. The output which is generated is fed to the next module which deals with the translation of the text in a given language into the final language. The working of this module is carried out using the Google Speech Recognition API. Python libraries, like the widely used SpeechRecognition library and Pyaudio library have been installed for this module. Pyaudio package is python support library that helps in accessing the microphone. The SpeechRecognition library has several inbuilt functions which are used to identify the background noises - like whispering, or people talking in the background, or loud footsteps, or construction noises, etc. - and cancel those ambient noises in order to focus on the foreground data which is basically the user-recorded speech. Finally, the filtered speech is converted into text.".

The second module of the system is Machine Translation module. This module deals with translation of the text which has been generated from the previous module into the language which it is supposed to translate. Before feeding the Neural Networks with the data output from the previous module of recognizing speech, data obtained is preprocessed. For preprocessing, the first task that is carried out is tokenization of the words for unique identification within a sentence. After tokenization, the sentences are padded so that all sentences become of the same length. The Machine Translation module of the system is totally based on the Recurrent Neural Network, which is used for translation purpose. The system deals with the basic Recurrent Neural Network, Recurrent Neural Network with Embedding, Bidirectional Recurrent Neural Network, and Recurrent Neural Network with embedded encoder and decoder. The work compares the accuracy for translations based on the model types of Recurrent Neural Network.

Published By:

Blue Eyes Intelligence Engineering \& Sciences Publication

(C) Copyright: All rights reserved.

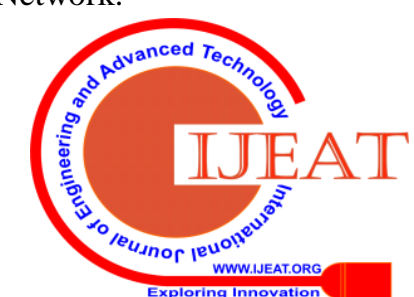


The output of the second module is taken as input for the third module which deals with synthesis of sentences. The last module is Speech Synthesis module. This is the final module, where the text - which is translated using several Recurrent Neural Network models, after interpreting the speech in source language - is converted back into speech in the destination language.

This is the part of the system where the machine speaks after translation is carried out from the speech in source language to the text generation in the destination language. Google Text to Speech API has been used for implementing this module of the system. The API provided by Google Text to Speech, supports the conversion of a text in a given language into speech in the same language.

\section{EXPERIMENTAL RESULTS}

The system has been built with several RNN models, namely the Basic Recurrent Neural Network model, Recurrent Neural Network with Embedding, Bidirectional Recurrent Neural Network, and Recurrent Neural Network with embedded encoder and decoder.

For visualizing the tabulated data, the python library matplotlib has been used for multiple line plot. Matplotlib is an open source python library which is widely used to deal with visualization of data in order to get better insight and analysis of the data. The system has been built over Google Colab which provides cloud services to execute codes, along with hardware supports including GPU.

Table-I: Accuracy percentage vs epoch for system models

\begin{tabular}{|l|l|l|l|l|}
\hline \multirow{2}{*}{$\begin{array}{c}\text { Epoch } \\
\text { Number }\end{array}$} & Basic RNN & $\begin{array}{c}\text { RNN with } \\
\text { Encoding }\end{array}$ & $\begin{array}{c}\text { Bidirectional } \\
\text { RNN }\end{array}$ & $\begin{array}{c}\text { RNN } \\
\text { Encoder } \text { and } \\
\text { Decoder }\end{array}$ \\
\hline 1 & 41.99 & 40.02 & 49.94 & 44.00 \\
\hline 2 & 47.07 & 44.49 & 59.04 & 49.78 \\
\hline 3 & 52.78 & 55.02 & 61.50 & 51.06 \\
\hline 4 & 56.98 & 64.14 & 63.10 & 53.01 \\
\hline 5 & 58.42 & 71.62 & 64.47 & 55.84 \\
\hline 6 & 58.82 & 76.32 & 65.38 & 57.24 \\
\hline 7 & 59.27 & 78.79 & 66.74 & 58.29 \\
\hline 8 & 60.12 & 80.58 & 67.23 & 59.24 \\
\hline 9 & 61.33 & 81.92 & 67.64 & 60.11 \\
\hline 10 & 62.07 & 83.18 & 68.08 & 60.77 \\
\hline
\end{tabular}

Table-I shows tabular comparison of accuracy percentages achieved for every round of the epoch for all of the models that are used in the system.

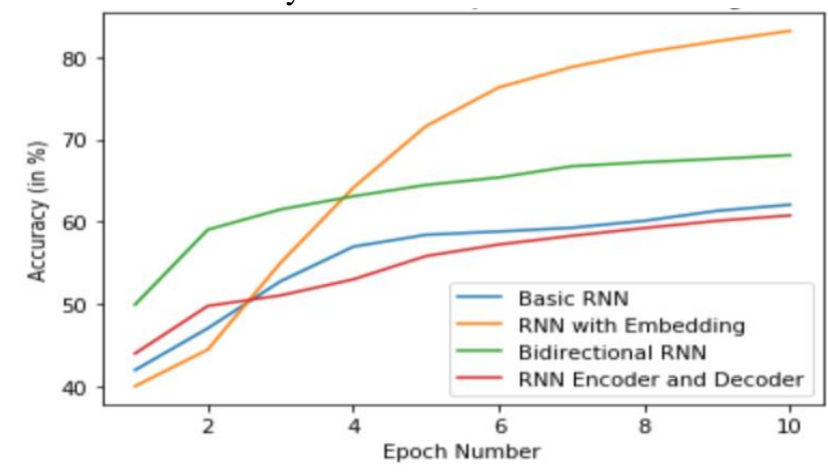

Fig. 2. Plot of accuracy vs. epoch number for models

The visualization of the data obtained through the plot shown in Fig. 2, where accuracy versus the iteration number of epochs is plotted in order to figure how the accuracy is increasing with every iteration for the four models. From the plot it can be clearly seen that the Recurrent Neural Network with encoding is having the highest accuracy with increase in iterations, bidirectional Recurrent Neural Network gives good amount of accuracy with time, and is more or less constant in nature, the same way, basic Recurrent Neural Network and Recurrent Neural Network with encoder and decoders also give kind of constant accuracy, but comparatively it is lower than the other two models.

The final system combines the top two models giving the highest accuracy, in order to boost up the overall accuracy of the system.

Table-II: Accuracy percentage vs epoch for new models

\begin{tabular}{|l|l|l|l|}
\hline \multirow{2}{*}{$\begin{array}{c}\text { Epoch } \\
\text { Number }\end{array}$} & \multicolumn{3}{|c|}{ Model Accuracy in \% } \\
\cline { 2 - 4 } & $\begin{array}{c}\boldsymbol{R} \boldsymbol{N} \text { with } \\
\text { Encoding }\end{array}$ & $\begin{array}{c}\text { Bidirectional } \\
\boldsymbol{R N N}\end{array}$ & $\begin{array}{c}\text { Bidirectional with } \\
\text { Encoding } \boldsymbol{R N N}\end{array}$ \\
\hline 1 & 40.02 & 49.94 & 53.29 \\
\hline 2 & 44.49 & 59.04 & 70.09 \\
\hline 3 & 55.02 & 61.50 & 79.89 \\
\hline 4 & 64.14 & 63.10 & 87.54 \\
\hline 5 & 71.62 & 64.47 & 93.27 \\
\hline 6 & 76.32 & 65.38 & 95.29 \\
\hline 7 & 78.79 & 66.74 & 96.12 \\
\hline 8 & 80.58 & 67.23 & 96.71 \\
\hline 9 & 81.92 & 67.64 & 97.35 \\
\hline 10 & 83.18 & 68.08 & 97.37 \\
\hline
\end{tabular}

Table-II shows the accuracy and its corresponding epoch iteration number for the new model along that combines Bidirectional and Encoding Recurrent Neural Network models with the other two tabular comparison of accuracy percentages achieved for every round of the epoch for all of the models that are used in the system.

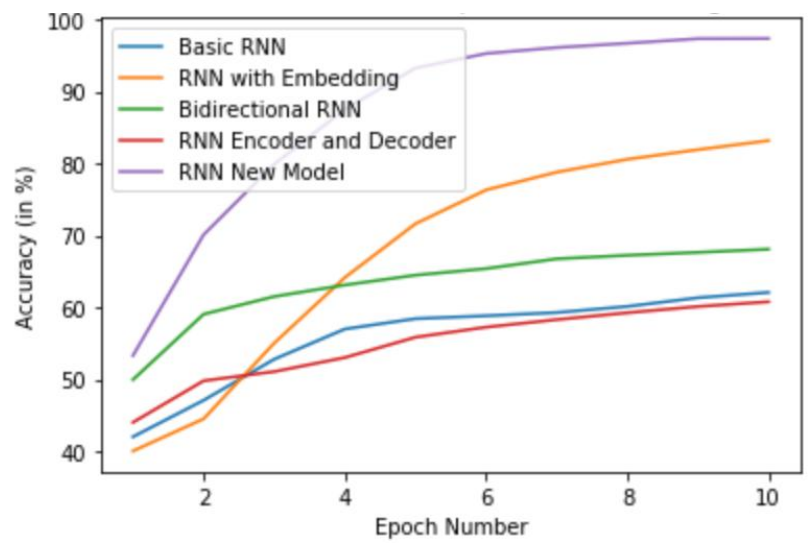

Fig. 3.Plot of accuracy vs. epoch number for new model

From the plot visualized in Fig. 3, the new RNN model that is created by merging of two previous RNN models, namely, bidirectional RNN and RNN with embedding, the accuracy is almost a cent percent.

\section{CONCLUSION}

The system built combines the models of Recurrent Neural Network giving the highest accuracies within ten epochs. It has been observed that taking multiple models at a time resulted in a better model giving higher accuracy for the system as a whole. It has also been observed that as individual models,

Published By:

Blue Eyes Intelligence Engineering

\& Sciences Publication

(C) Copyright: All rights reserved.

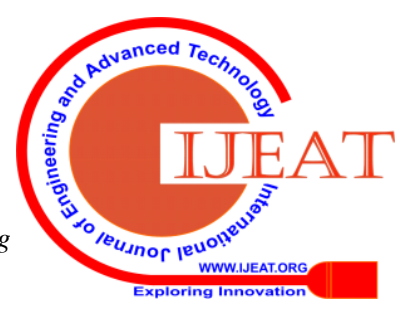


Recurrent Neural Network with embedding gives the maximum accuracy with further iterations, followed by bidirectional Recurrent Neural Network. The least performing individual models include the basic Recurrent Neural Network and the Recurrent Neural Network.

The model must be chosen smartly, and while combining, it should be taken care that the system must not result to overfitting issues and other overheads, as they will lead to worse accuracy and an inefficient system for machine translation using neural networks. According to the results observed from the experiments done, bidirectional Recurrent Neural Network with encoding algorithm gives a high accuracy compared to that of the given models of Recurrent Neural Network. Single Recurrent Neural Network models like the basic RNN, only RNN with encoding, only bidirectional RNN and RNN with encoder and decoder gives lesser accuracy comparatively.

The work can be extended for a larger dataset consisting of even bigger text corpus, so that the system becomes ideal for translating every possible sentence instead of just a few sentences. Further, the possible combination of models can be broadened into other models of RNN as well, considering all possible combinations within them, so that the systems can be made further accurate.

\section{ACKNOWLEDGMENT}

We would like to thank the faculties belonging to the School of Computer Science and Engineering (SCOPE) of Vellore Institute of Technology, who have cooperated and helped us throughout our research. We are also grateful to our fellow students and other staff for dedicating their time and support for successful completion of the research.

\section{REFERENCES}

1. Shadiev, R., Hwang, W. Y., Chen, N. S., \& Huang, Y. M. (2014) Review of speech-to-text recognition technology for enhancing learning. Journal of Educational Technology \& Society, 17(4), 65-84.

2. Soltau, H., Liao, H., \& Sak, H. (2016). Neural speech recognizer: Acoustic-to-word LSTM model for large vocabulary speech recognition. arXiv preprint arXiv:1610.09975.

3. Xiong, W., Droppo, J., Huang, X., Seide, F., Seltzer, M., Stolcke, A., .. \& Zweig, G. (2016). Achieving human parity in conversational speech recognition. arXiv preprint arXiv:1610.05256.

4. Shang, L., Lu, Z., \& Li, H. (2015). Neural responding machine for short-text conversation. arXiv preprint arXiv:1503.02364.

5. Battenberg, E., Chen, J., Child, R., Coates, A., Li, Y. G. Y., Liu, H., ... \& Zhu, Z. (2017, December). Exploring neural transducers for end-to-end speech recognition. In 2017 IEEE Automatic Speech Recognition and Understanding Workshop (ASRU) (pp. 206-213). IEEE.

6. Bahdanau, D., Cho, K., \& Bengio, Y. (2014). Neural machine translation by jointly learning to align and translate. arXiv preprint arXiv:1409.0473.

7. Luong, M. T., Pham, H., \& Manning, C. D. (2015). Effective approaches to attention-based neural machine translation. arXiv preprint arXiv:1508.04025.

8. Cho, K., Van Merriënboer, B., Gulcehre, C., Bahdanau, D., Bougares, F., Schwenk, H., \& Bengio, Y. (2014). Learning phrase representations using RNN encoder-decoder for statistical machine translation. arXiv preprint arXiv:1406.1078.

9. Cho, K., Van Merriënboer, B., Bahdanau, D., \& Bengio, Y. (2014). On the properties of neural machine translation: Encoder-decoder approaches. arXiv preprint arXiv:1409.1259.

10. Sennrich, R., Haddow, B., \& Birch, A. (2015). Neural machine translation of rare words with subword units. arXiv preprint arXiv:1508.07909.

\section{AUTHORS PROFILE}

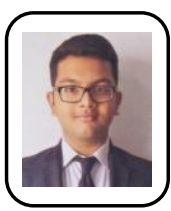

Debajit Datta is currently a third-year undergraduate pursuing Computer Science and Engineering from Vellore Institute of Technology, Vellore. He is an experienced front-end developer with experience of working in the non-profit organization management industry. He has been an active member of the clubs and chapters in the university and has participated is several hackathons at university level. He has been an active member of Developers Student Clubs VIT, a student chapter powered by Google Developers Group and Venturesity VIT, a student chapter. He has industrial exposures from five different industrial internships within the three years of B. Tech. He has also presented a research paper at ic-ETITE'20 conference organized by IEEE and supported by ACM.

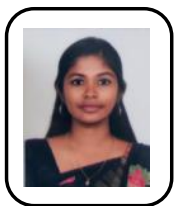

Dr. Preetha Evangeline David is currently working as an Assistant Professor (Senior grade) in the School of Computer Science at Vellore Institute of Technology, Vellore, India. She holds a PhD from Anna University, Chennai in the area of Cloud Computing. She has published many research papers in highly reputed journals. Her current research focuses on Artificial Intelligence, Digital Twin Technology, High Performance Computing, Computational Intelligence and Data Structures. Her upcoming research would be Multi-disciplinary in collaboration with various departments to solve socially related challenges and provide solutions to human problems.

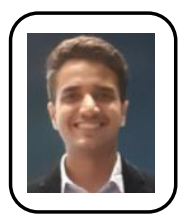

Dhruv Mittal is currently studying in Vellore Institute of Technology, Vellore. He is a third-year student pursuing Bachelors of Technology in the field of Computer Science. $\mathrm{He}$ is keen to learn about new technologies and advancement in the field of computers. He has been actively participating in various Hackathons and meetups in order to explore new opportunities and learn about upcoming technologies. He has been an active member of Developers Student Clubs VIT, a student chapter powered by Google Developers Group.

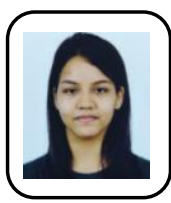

Anukriti Jain is currently pursuing bachelors in Computer Science and Engineering from Vellore Institute of Technology, Vellore. She is a third-year undergraduate who has been an active member of several clubs and chapters of the college. She is a member of VIT IEEE Computer Society, a student chapter and is an active determined and hardworking
Blue Eyes Intelligence Engineering \& Sciences Publication 